\title{
Combined Intranasal Insulin/Saxagliptin/Metformin Therapies Ameliorate the Effect of Combined Oral Contraceptive- (COC-) Induced Metabolic Syndrome (MetS) with a Major Target on Glucose Metabolism in Adult Female Wistar Rats
}

\author{
Saheed Olanrewaju Afolabi $(\mathbb{D}$, Joy Folahan, Olalekan Agede, and Olufunke Olorundare \\ Department of Pharmacology and Therapeutics, University of Ilorin, Ilorin, Nigeria \\ Correspondence should be addressed to Saheed Olanrewaju Afolabi; afolabi.so@unilorin.edu.ng
}

Received 10 June 2021; Revised 12 September 2021; Accepted 12 November 2021; Published 13 December 2021

Academic Editor: Vikas Roy

Copyright (c) 2021 Saheed Olanrewaju Afolabi et al. This is an open access article distributed under the Creative Commons Attribution License, which permits unrestricted use, distribution, and reproduction in any medium, provided the original work is properly cited.

\begin{abstract}
Objective. To evaluate the effect of the chronic use of combined oral contraceptives (COCs: ethinyl estradiol and levonorgestrel) on the indices of metabolic syndrome in adult female Wistar rats and possible therapeutic management. Materials and Methods. 64 female Wistar rats received either distilled water, norethindrone (NOR), COC, intranasal insulin (INI), metformin (MET), saxagliptin (SAX), INI+MET, and INI+SAX. After 8 weeks of exposure to COC, the animals were sorted into the therapeutic groups. Several parameters were assayed for, such as body weight changes, fasting blood glucose (FBG) level, insulin levels, inflammatory cytokines, and glycated hemoglobin (Hb1Ac). Results. The levels of FBG, insulin, and Hb1Ac were increased consequent upon COC treatment. Treatment with INI+SAX and INI+MET reduced significantly the levels of FBG and Hb1Ac; in addition, the level of insulin was significantly increased in the INI+MET groups $(p \leq 0.05)$. Serum lipid profile analysis showed a statistical reduction in high-density lipoprotein (HDL) level; this reduction was also significantly reversed in the INI+ SAX group. Reduced catalase activity observed in the COC group was reversed in the INI+MET group ( $p \leq 0.05)$. A nonsignificant increase in the level of TNF- $\alpha$ as a result of COC treatment was reversed by INI and INI+MET treatment. Liver GLUT4 and G-6-phosphate levels were significantly increased by COC treatment, and this effect was reversed by INI+SAX in both assays, respectively $(p \leq 0.01)$. Conclusions. The use of MET and SAX in combination with INI has been shown to reverse some indices of MetS. This study proposes a clinical phase to backup and ascertain these preclinical findings.
\end{abstract}

\section{Introduction}

Premenopausal women are active users of hormonal contraceptives. In the last decade, there has been an upsurge in this usage; scientific reports have estimated that over 150 million sexually active women subscribe to these pharmaceuticals globally [1-3]. Commercially available formulations consisting of a synthetic estrogen and progestin constitute the globally recognized combined oral contraceptives (COCs) $[4,5]$. In addition to its contraceptive effects, constant administration of COC is also being explored as a therapeutic intervention against polycystic ovary syndrome (PCOS) and dysmenorrhea $[6,7]$. However, the use of COC has been linked with disruption in body weight regulation, arterial blood pressure, glucose metabolism, and general homeostatic regulations in females $[8,9]$. Endocrinologists and researchers in the field of reproductive health suggest that continuous prescription of COC should be revised with respect to these adverse effects $[10,11]$. Ethinyl estradiol (EE), synthesized from $17 \beta$-estradiol (E2), is the most used synthetic estrogen in COC $[7,12,13]$; it is an estrogen receptor agonist. Over the years, ethinyl estradiol has been used for menopausal symptoms, gynecological disorders, and some forms of hormone-sensitive cancers [14-16]. Currently, EE is almost exclusively employed for the adjunctive role it plays in the COC, making it the most widely used 
estrogen [17]. Levonorgestrel (LEV) is a use-alone emergency contraceptive pill and is also formulated with estrogen in some COC [18]. It is a progestin that works principally by preventing ovulation and enhancing the closure of the cervix to prevent the entry of sperm, similar to the hormone progesterone $[19,20]$. LEV is a synthetic steroid (17 $\alpha$-ethynyl-18methyl-19-nortestosterone) derived from testosterone [21].

The combination of these hormones has been studied for their therapeutic efficacy in women who have PCOS, a pathological condition characterized by dysregulation in and carbohydrate metabolism in women [22, 23]. Generally, COCs from its inception have been linked with dysregulation of glucose homeostasis. The COCs containing norethindrone and EE have reportedly increased glycemia levels in premenopausal women [24]; this effect was in tandem with COC containing levonorgestrel and EE [25].

Glycemic regulation is under the control of the hormone, insulin. When glucose level in the extracellular fluid increases, it is transported to the pancreatic $\beta$-cells and its consequent metabolism raises the intracellular ATP/ADP ratio. This closes the ATP-sensitive/K+ channels (KATP), causing depolarization of the $\beta$-cell membranes and activation of $\mathrm{Ca}^{2+}$-activated voltage channels; the $\mathrm{Ca}^{2+}$ influx then activates the release of insulin granules via exocytosis [26, 27]. Insulin resistance (IR) is a metabolic disorder that features a decreased response to both exogenous and endogenous insulin in cells, specific adipose tissues, skeletal muscles, and the whole body. IR is one of the major indicators and pathological driver of metabolic syndrome, a disease condition caused by a myriad of metabolic mishaps which features IR, hyperinsulinemia, glucose intolerance, and dyslipidemia [28, 29]. Metabolic syndrome is suggested to be the most important medical problem of the 21 st century. Insulin resistance is a known risk factor for type 2 diabetes [30, 31]. Metformin is an oral hypoglycemic agent belonging to the biguanide class of antidiabetic agents. It is often used as first-line treatment of diabetes [32, 33]. It improves hyperglycemia primarily through its suppression of hepatic glucose production, increases insulin sensitivity, enhances peripheral glucose uptake, upregulates oxidation of fatty acid [34], and downregulates glucose absorption from the gastrointestinal tract. A member of the dipeptidyl peptidase-4 (DPP-4) inhibitor group of oral hypoglycemics, saxagliptin is approved by the US Food and Drug Administration and the European Medicines Agency. DPP-4 inhibitors are believed to be therapeutically effective in the management of IR and thus could prevent metabolic syndrome [35].

Here, we set out to investigate the effect of combined oral contraceptives (ethinyl estradiol and levonorgestrel) and minipill (norethindrone) on adult female albino rats; and the possible ameliorative effect of single/combined administration of saxagliptin, intranasal insulin and metformin on the metabolic syndrome status.

\section{Materials and Methods}

2.1. Experimental Groups. Adult female Wistar rats (64) were obtained from an animal house facility in Ogbomosho,
Oyo state, Nigeria, and housed in groups of eight and allowed to acclimatize for 7 days in the animal housing facility of the Faculty of Basic Medical Sciences, University of Ilorin, Nigeria. Housing conditions were temperature of $25^{\circ} \mathrm{C}$ and a $12: 12$ light: dark cycle with light on by $07: 00$ and off at 1900. The animals were housed in metabolic cages with wood shavings as beddings replaced fortnightly. Animals had unrestricted access to rat pelletized feed from Top Feed Nigeria Ltd. (consisting of maize, soybean, GNC, corn brown, wheat offal, palm kernel cake, and bone) and water ad libitum. Animals were monitored and cleaned up daily. All eight groups received distilled water/NOR/COC for 8 weeks; then, each group received various therapeutic regimen for 7 days as outlined below. All protocols involving the use of experimental animals were duly approved by the University Ethical Committee with the approval number UERC/ASN/2020/2028. The international guide to handle and use of experimental animals by the National Research Council [36] and Committee for the Update of the Guide for the Care and Use of Laboratory Animals [37] was strictly adhered to during the animal handling phase of this study.

The animal experimental groupings are underlisted.

Group 1. Control

Group 2. Norethindrone (NOR), $1.4 \mathrm{mg} / \mathrm{kg} /$ day

Group 3. Combined oral contraceptives (EE and LEV; COC), $7.6 \mathrm{mg} / \mathrm{kg} /$ day

Group 4. COC+intranasal insulin (INI: 2 IU, $1 \mathrm{IU} / 10 \mu \mathrm{l}$ and $10 \mu \mathrm{l} /$ nostril)

Group 5. COC+metformin (MET: $150 \mathrm{mg} / \mathrm{kg} /$ day)

Group 6. COC+saxagliptin (SAX: $10 \mathrm{mg} / \mathrm{kg} / \mathrm{day}$ )

Group 7. COC+INI+MET

Group 8. COC+INI+SAX

2.2. Drugs. Recombinant human insulin (actrapid, $100 \mathrm{IU} /$ $\mathrm{ml}$ ) was purchased from Novo Nordisk, combined oral contraceptives (ethinyl estradiol and levonorgestrel) were from Bayer USA, and metformin, norethindrone (minipill), and saxagliptin were purchased from MedPlus pharmacy Ilorin. All biochemical reagents including ELISA kits for rat insulin, glucose-6-phosphate, lipids, and glucokinase were purchased from Elabscience (US). All other chemicals were of analytical grade and commercially available. Accu-Chek glucometer and glucose strips were purchased from Roche Diagnostic, USA (Micropipette P-10, Eppendorf).

2.3. Intranasal Insulin Administration. According to the method described by Njan et al. [38], rats were anesthetized by placing them in tightly sealed transparent glass jars containing isoflurane (5\%) obtained from Southern Anesthesia \& Surgical, (West Columbia, US) for brief periods $(<30 \mathrm{~s})$, removed, and restrained in the supine position. A total of $2 \mathrm{IU}(1 \mathrm{IU} / 10 \mu \mathrm{l}$ and $10 \mu \mathrm{l} /$ nostril) of rapid acting insulin or vehicle $(0.9 \%$ Sodium Chloride, $\mathrm{NaCl})$ was rapidly dispensed intranasally using a micropipette (P-10, Eppendorf). This procedure lasted for about $15 \mathrm{~s}$. Animals became conscious in another 15-20s and were observed physically before returning to the metabolic cages. 


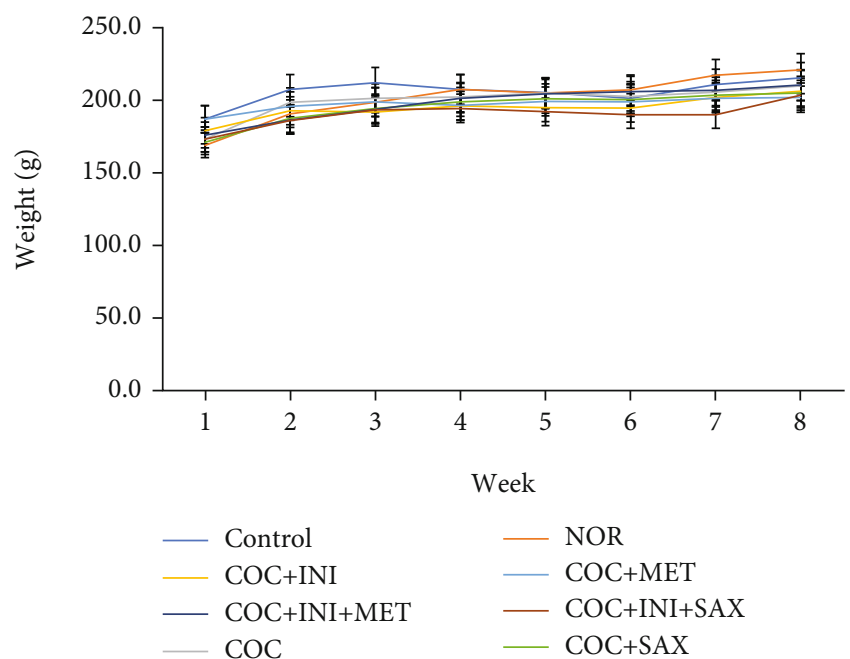

(a)

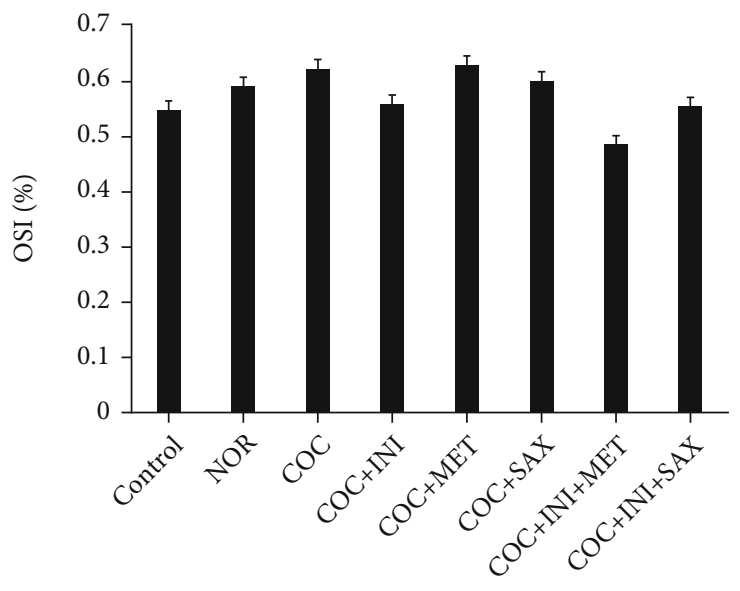

(b)

FIGURE 1: (a) The effect of treatment groups on weekly body weight monitoring. There was no statistically significant alteration in the weight of the treatment groups when compared with the control at $p \leq 0.05$; mean \pm SEM, $n=8$. (b) The organosomatic index (OSI) carried out on the pancreas showed no statistical difference in the weight of the organ in the treatment group when compared with the control $p \leq 0.05$; mean \pm SEM, $n=8$.

2.4. Measurement of Body Weight, Organosomatic Index, Fasting Blood Glucose, and Glucose Tolerance Test. Body weights of control and experimental rats were analyzed weekly throughout the experimental phase. At the end of the experimental period, the animals were sacrificed, the pancreas was harvested, and the organosomatic index was calculated (i.e., weight of the pancreas as a percentage of the animal body weight). Fasting blood glucose levels were analyzed using a glucometer (Accu-Chek Performa, Roche Diagnostic, USA). An oral glucose tolerance test (OGTT) was performed using an oral dose of glucose $(2 \mathrm{~g} / \mathrm{kg})$ for $2 \mathrm{~h}$ after 8 consecutive weeks of treatment. Prior to the OGTT procedure, animals were fasted overnight and given only water to drink throughout the time. The blood samples were collected from each group just before glucose administration $(0 \mathrm{~min})$ and at 30,60 , and $120 \mathrm{~min}$ after glucose administration.

2.5. Plasma and Serum Biochemical Assays. Albino rats were sacrificed using isoflurane $(5 \% \mathrm{~W})$ anesthesia, and blood was collected by cardiac puncture. Serum was isolated by centrifugation (LABCENT 5000) at $3000 \mathrm{rpm} 15 \mathrm{~min}$ as per standard protocols. Serum insulin, C-peptide, C-reactive protein (CRP), and Adenosine Deaminase (ADA) levels were assayed by the solid-phase enzyme-linked immunosorbent assay (ELISA) (Millipore; Billerica, USA, cat. no.: EZRMI-13K). Method described by Wilson and Spiger [39] was used to determine the lipid profile. Lipoproteins were fractionated by a dual precipitation technique of and highdensity lipoprotein (HDL) and low-density lipoprotein (LDL) [40]. Total cholesterol (TC) and triglycerides (TGs) were measured using commercially available kits (Abcam, UK) following the manufacturer's protocol. Glycosylated hemoglobin (HbA1C) levels were estimated by the method described by Rao and Pattabiraman [41].

2.6. Pancreatic and Liver Homogenate Assays. Liver and pancreas homogenates, $10 \%(\mathrm{w} / \mathrm{v})$, were prepared in a potassium phosphate buffer $0.05 \mathrm{M}, \mathrm{pH}$ 7.4. Elvehjem homogenizer (Dominique Dutscher, Issy-les-Moulineaux, France) was used. The homogenates were centrifuged for $10 \mathrm{~min}$ at $3000 \times \mathrm{g}$ at $4^{\circ} \mathrm{C}$, and the supernatant aliquots were saved and stored at $-20^{\circ} \mathrm{C}$. The homogenate protein content was measured according to the method described by Lowry et al. [42]. Superoxide dismutase (SOD) activity was measured by rate of inhibition of pyrogallol autooxidation on a spectrophotometer at $470 \mathrm{~nm}$ according to the method of Marklund and Marklund [43]. SOD activity was expressed as units per milligram protein. The method described by Rotruck et al. was used to determine the glutathione peroxidase (GPx) level. It was calculated by the rate of NADPH oxidization spectrophotometrically at $340 \mathrm{~nm}$ [44]. GPx activity was presented as micrograms $(\mu \mathrm{g})$ of reduced glutathione (GSH) expended/ $\mathrm{min} / \mathrm{mg}$ protein. The method described by Sinha was used to quantify the catalase (CAT) activity as estimated by monitoring the decomposition of $\mathrm{H}_{2} \mathrm{O}_{2}$ spectrophotometrically at $240 \mathrm{~nm}$ [45]. Levels of tumor necrosis factor-alpha (TNF- $\alpha$; cat. no.: CSB-E04741m), interleukin-1 beta (IL-1 $\beta$; cat. no.: CSB-E04621m). and interleukin-6 (IL-6; cat. no.: CSB$\mathrm{E} 04627 \mathrm{~m}$ ) were assessed in the pancreas supernatant by ELISA using anti-mouse antibody (CUSABIO Life Sciences, Wuhan, China) based on the manufacturer's instructions.

Liver xanthine oxidase level was determined using methods described by Mohamed et al. [46]; membrane GLUT4 transporters were quantified using ELISA kit procured from Life Science Inc. (USA) following the manufacturer's 


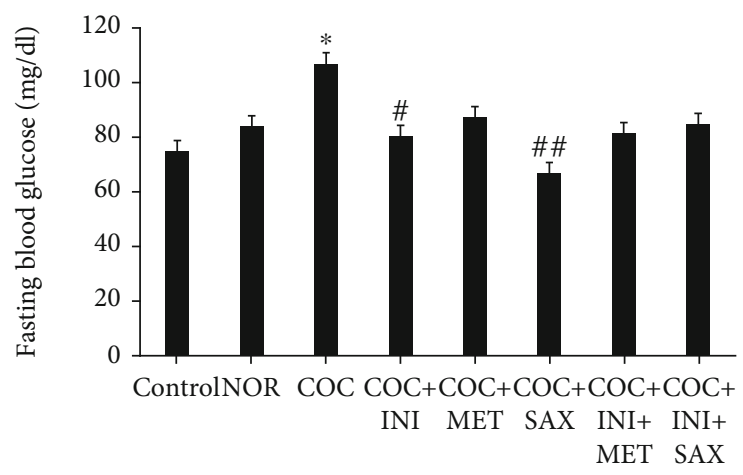

(a)

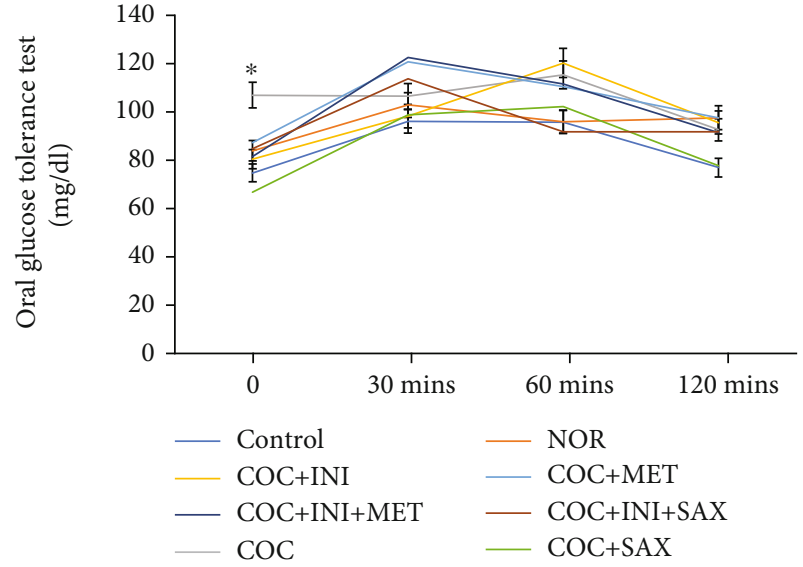

(b)

Figure 2: (a) COC caused a significant increase in the fasting blood glucose level when compared with control $\left({ }^{*} p \leq 0.05\right)$; this increase was significantly reversed by administration of SAX $\left({ }^{\# \#} p \leq 0.01\right)$. INI administration also reduced the FBG significantly $\left({ }^{\#} p \leq 0.05\right)$; mean \pm SEM, $n=8$. (b) The oral glucose tolerance test showed an initial increase in the blood glucose. However, there was no significant effect on the tolerance test among the tested group in the course of the 120 mins tolerance test; mean \pm SEM, $n=6$.

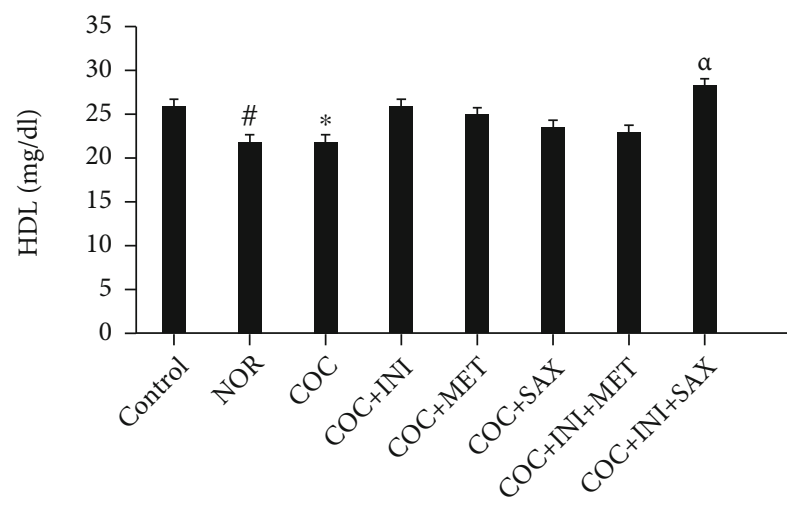

(a)

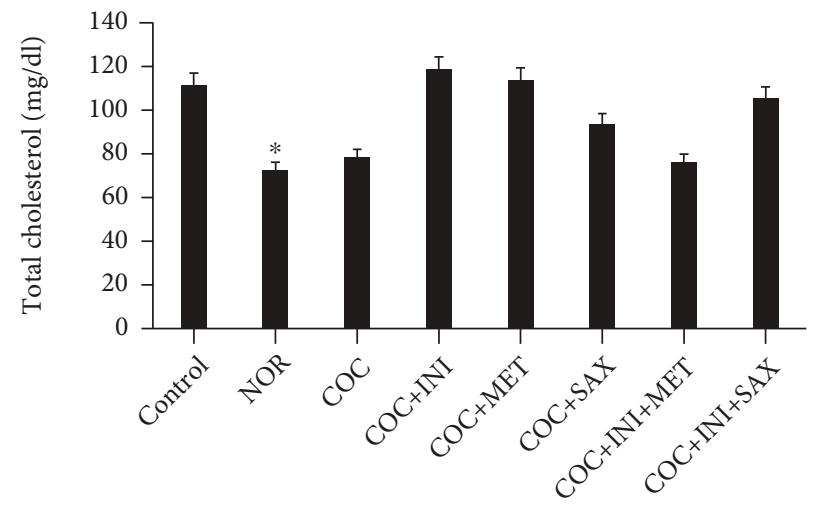

(c)

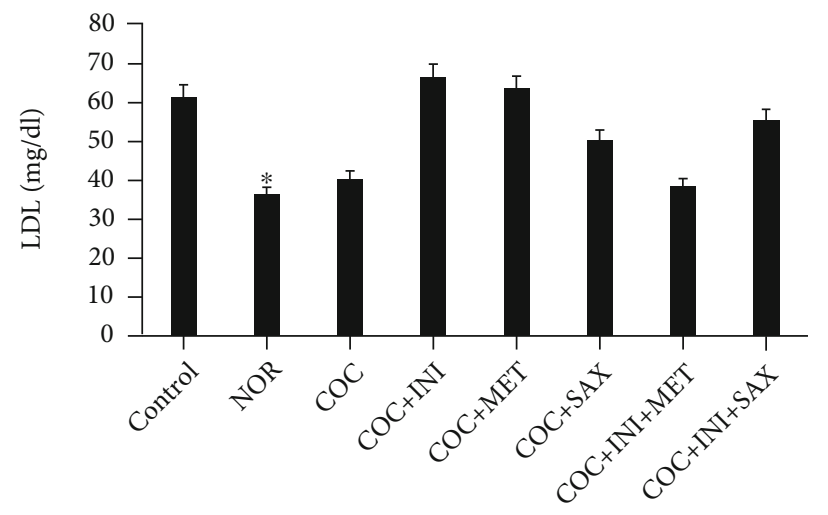

(b)

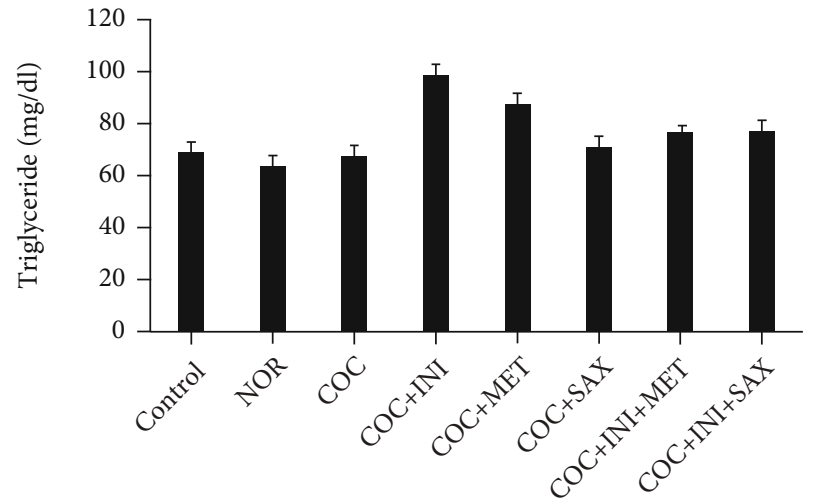

(d)

Figure 3: (a) COC caused a significant decrease in HDL level when compared with control $\left({ }^{*} p \leq 0.05\right)$; this was also observed in the NOR group ( $\left.{ }^{*} p \leq 0.05\right)$. INI+SAX significantly $\left({ }^{\alpha} p \leq 0.05\right)$ increased the HDL level when compared with the COC group. (b, c) A significant reduction in the LDL and total cholesterol levels of the NOR-treated group, respectively $\left({ }^{*} p \leq 0.05\right)(\operatorname{mean} \pm \operatorname{SEM}, n=8)$.

instructions [47]; glucokinase (GCK) level was quantified using the ELISA method in accordance with the instructions of the GCK ELISA kit (provided by Beijing Lvyuan Bode Biotechnology Co., Ltd.)
2.7. Statistical Analysis. Results are presented as means \pm SEM, one-way ANOVA, parametric (unpaired Student's $t$-test), or nonparametric (Mann-Whitney $U$-test) unpaired tests also using GraphPad Prism ${ }^{\circledR}$ version 6.00 for Windows 


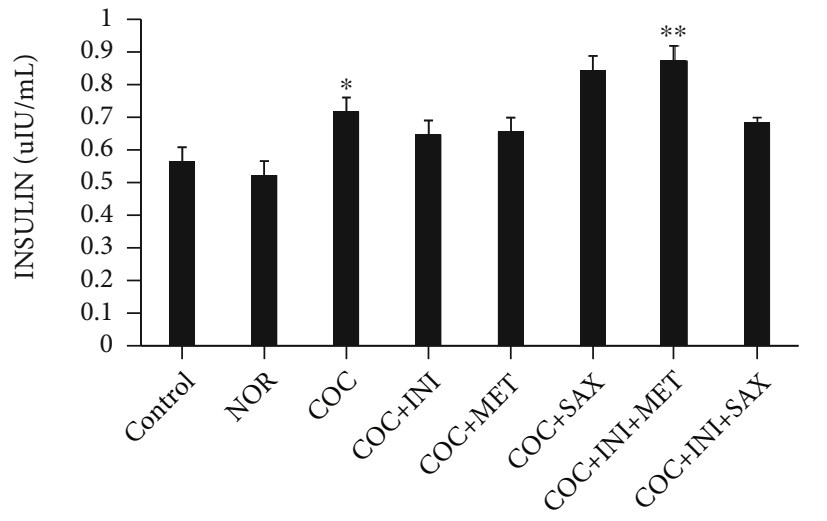

(a)

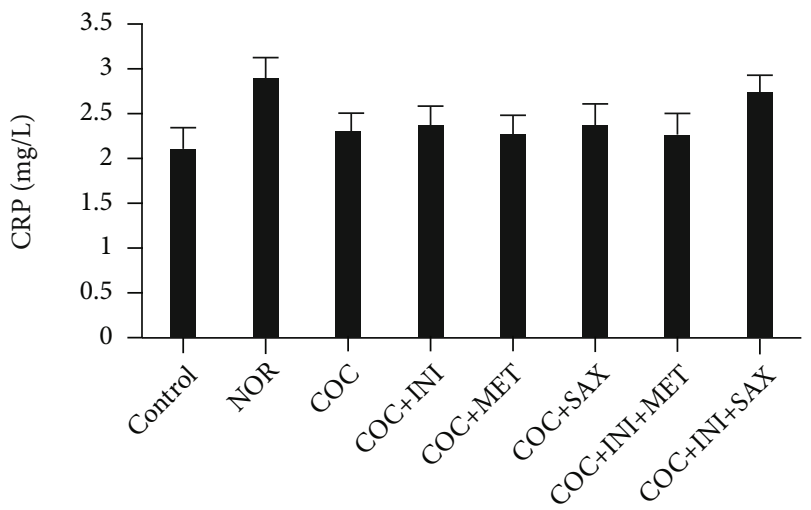

(c)

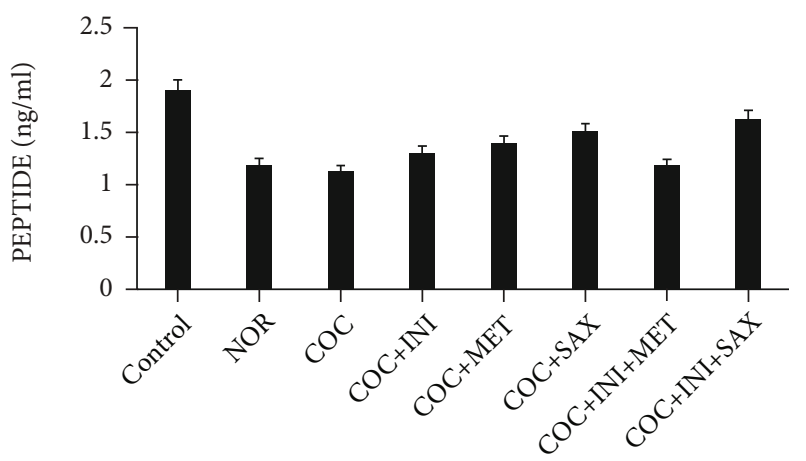

(b)

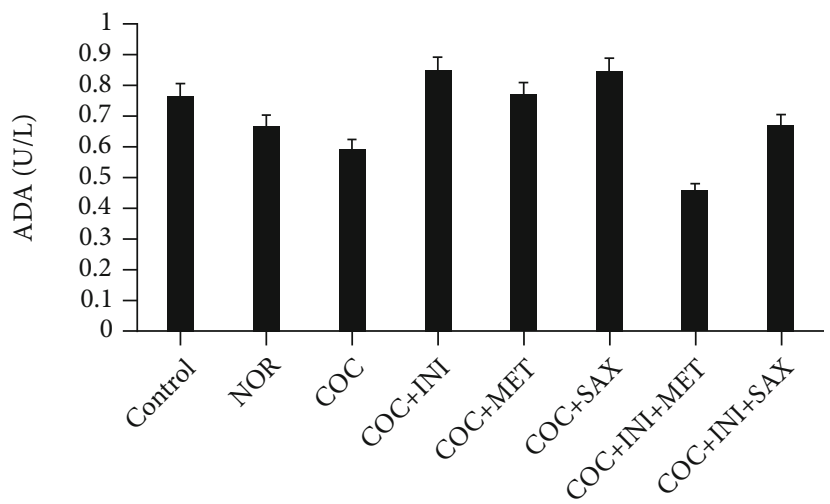

(d)

Figure 4: (a) COC caused a significant increase in insulin level when compared with control $\left({ }^{*} p \leq 0.05\right)$; this was also observed in the INI+MET group $\left({ }^{* *} p \leq 0.01\right)$ when compared with the COC group. (b, c) No significant difference across the groups for C-peptide and $\mathrm{CRP}$, respectively (mean $\pm \mathrm{SEM}, n=8$ ).

(San Diego, CA, USA). The level of significance was set at $p$ $<0.05$.

\section{Results}

3.1. Body Weight and Pancreatic Organosomatic Index Evaluation. The 9-week study featured a weekly monitoring of the animal weight per group $(n=8)$. There was no statistically significant difference across the treatment group (Figure 1(a)). This trend was similar in the organosomatic index, which also showed no significant alteration in this index across the experimental groups (Figure 1(b)).

\subsection{Fasting Blood Glucose Level and Oral Glucose Tolerance} Test. There was a significant increase in the fasting blood glucose level of the COC group $(p \leq 0.05)$; this alteration was however reversed in the INI group $(p \leq 0.05)$ and the SAX group $(p \leq 0.01)$ (Figure $2(a))$. The OGTT showed no significant effect across the treatment groups except for the initial spike in glucose level observed at the 0 min timepoint (Figure 2(b)).

3.3. Serum Lipid Profile Analysis. HDL levels were significantly reduced in both COC $(p \leq 0.05)$ and NOR $(p \leq 0.05)$ groups. This reduction was reversed in the INI+SAX group $(p \leq 0.05)$ (Figure 3(a)). The NOR group showed a significant reduction in both LDL and total cholesterol levels $(p \leq 0.05)$ (Figures 3(b) and 3(c), respectively). There was no significant alteration in the triglyceride level across the experimental groups.

3.4. Evaluation of Insulin, C-Peptide, C-Reactive Protein, and $A D A$ Levels. Insulin level was significantly increased in the COC group $(p \leq 0.05)$ when compared with the control; this level was further increased in the INI+MET group $(p \leq 0.01)$ (Figure 4(a)). The C-peptide, C-reactive protein, and ADA levels were not significantly different across the experimental groups (Figures 4(b)-4(d), respectively).

3.5. Assessment of Antioxidant Parameters on Pancreas Homogenate. Catalase level was significantly reduced in the COC group when compared with the control $(p \leq 0.05)$; this reduction was reversed in the INI+MET group $(p \leq 0.01)$. SOD, GPx, and GSH levels were not significantly affected across the experimental groups (Figures 5(b)-5(d)); total protein and MDA levels were not also significantly affected across the experimental groups (Figures 6(a) and 6(b)).

3.6. Assessment of TNF- $\alpha, I L-6$, and IL-1 $\beta$ Levels in Pancreas Homogenate. Figures $7(\mathrm{a})-7$ (c) show that levels of TNF- $\alpha$, 


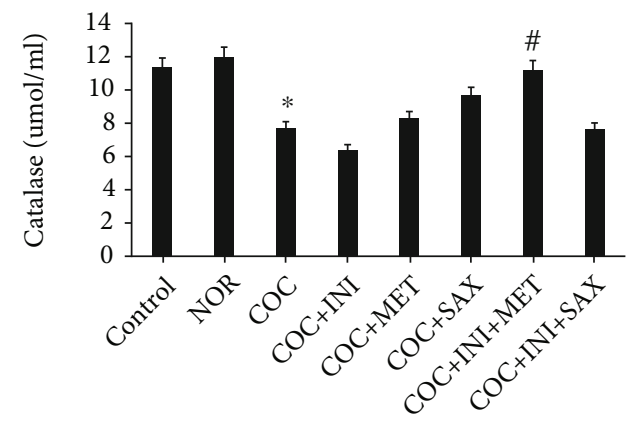

(a)

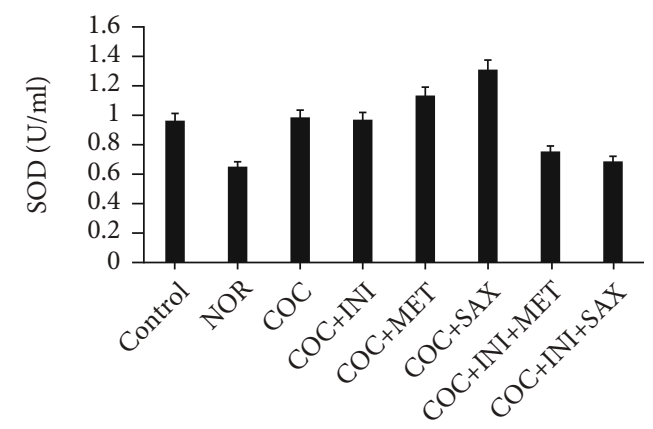

(c)

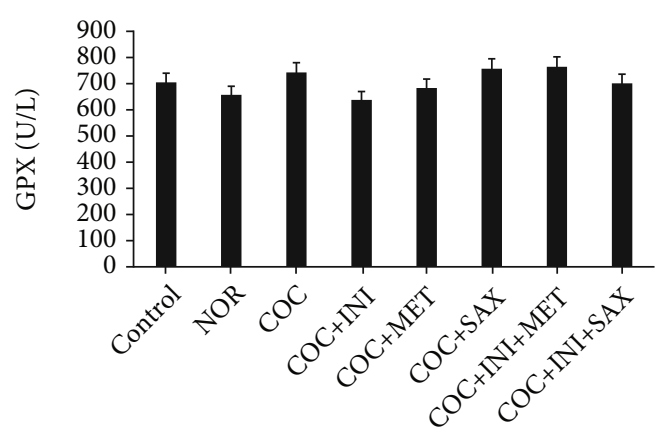

(b)

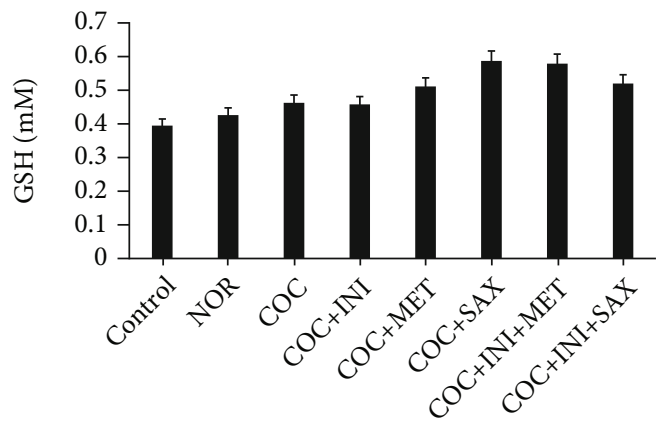

(d)

FIGURE 5: (a) COC caused a significant decrease in catalase level when compared with control $\left({ }^{*} p \leq 0.05\right)$; this decrease was reversed in the INI+MET group $\left({ }^{\#} p \leq 0.01\right)$ when compared with the COC group. (b, c) No significant difference across the groups for SOD and GPX, respectively (mean \pm SEM, $n=6$ ).

IL-6, and IL- $1 \beta$ were not significantly different statistically in comparison with the control. However, nonstatistical compares suggest an increase in the TNF- $\alpha$ levels in the COC group and a subsequent reduction in the MET and INI+ MET groups.

3.7. Evaluation of Glucokinase, G6P, and GLUT4 Levels in Liver Homogenate. COC caused a significant decrease in glucokinase level when compared with control $\left({ }^{* *} p \leq 0.01\right)$; this decrease was not reversed by any of the treatment group when compared with the COC group (Figure 8(a)). COC increased the G6P level $\left({ }^{* *} p \leq 0.01\right)$. This effect was reversed significantly by the INI+SAX group $\left({ }^{\# \#} p \leq 0.01\right)$ (Figure $8(\mathrm{~b})$ ). COC increased the GLUT4 levels significantly $\left({ }^{* *} p \leq 0.01\right)$; this effect was reversed in INI+MET $\left({ }^{*} p \leq 0.05\right)$ and INI+SAX $\left({ }^{\# \#} p \leq 0.01\right)$ (Figure 8(c)).

3.8. Evaluation of Plasma HbAlc and Liver Xanthine Oxidase Levels. Glycated hemoglobin (HbA1c) levels assayed for in the plasma showed a significant increase in the COC group $\left({ }^{*} p \leq 0.05\right)$; this effect was reversed significantly in the MET, SAX, and INI+MET groups $\left({ }^{* *} p \leq 0.01\right)$ (Figure 9(a)). Xanthine oxidase levels were not significantly different across the experimental groups (Figure 9(b)).

\section{Discussion}

A constellation of metabolic abnormalities which features hyperglycemia, hyperinsulinemia, and hyperlipidemia among other disorders was initially termed as "Insulin resistance syn- drome" [48]. These metabolic abnormalities, generally now referred to as metabolic syndrome (MetS), are increasing at an alarming rate, becoming a major public and clinical problem worldwide [49]. MetS has been linked to cardiovascular dysfunction and a major risk factor in the development of type 2 diabetes mellitus [50,51]. This present study sets out to unravel the effect of combined oral contraceptives (COCs) on specific elements of MetS and the possible benefits of intranasal insulin (INI), metformin (MET), and saxagliptin (SAX) in various combination regimens in the management of this syndrome. It is expected that the results from this study shed light on the underlying therapeutic intervention for this disorder occasioned by a chronic administration of COC in adult female albino Wistar rats.

Body weight changes and particularly body mass index (BMI) have been linked directly to metabolic syndrome status in humans [52], with a particular interest in the relationship between MetS and waist circumference [53, 54]. Weekly monitoring of the experimental groups showed that the chronic exposure to combined oral contraceptives did not affect the animal body weight during the study. In their study, Ghezzi et al. postulated that weight loss or distortion takes place in the early developmental stages of the animals, and this study attributed the unlikelihood of weight gain at maturity to a physiological decline in the development of the animals at adulthood [55]. This phenomenon is in tandem with our findings, since adult female Wistar rats were used. Organosomatic index of the pancreas was also in line with the body weight changes, and this is expected due to the fact that insulin resistance may not be a sign of toxicity 


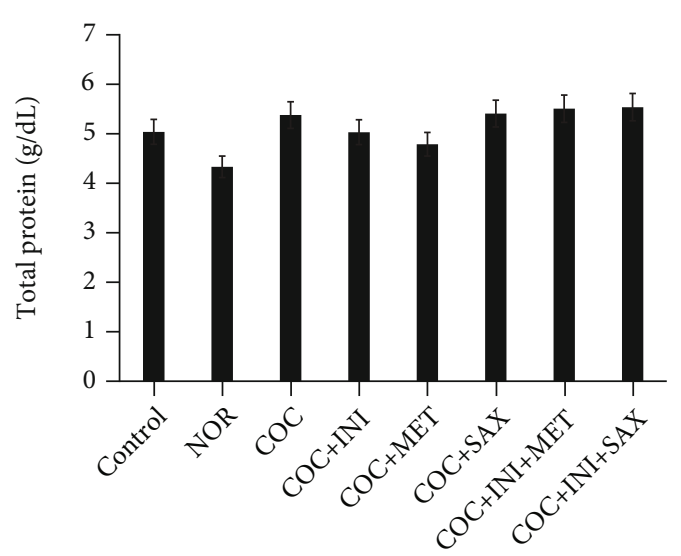

(a)

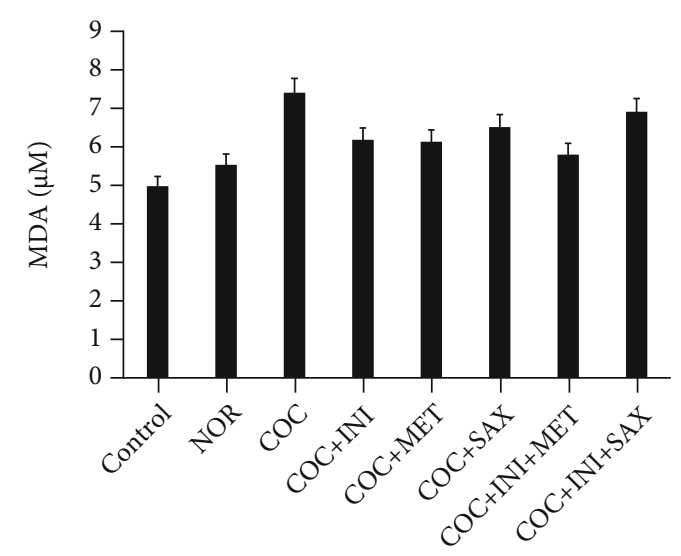

(b)

FIgURE 6: $(\mathrm{a}, \mathrm{b})$ No significant alterations across the test groups for total protein and MDA levels, respectively; $p \leq 0.05 ;$ mean \pm SEM, $n=6$.

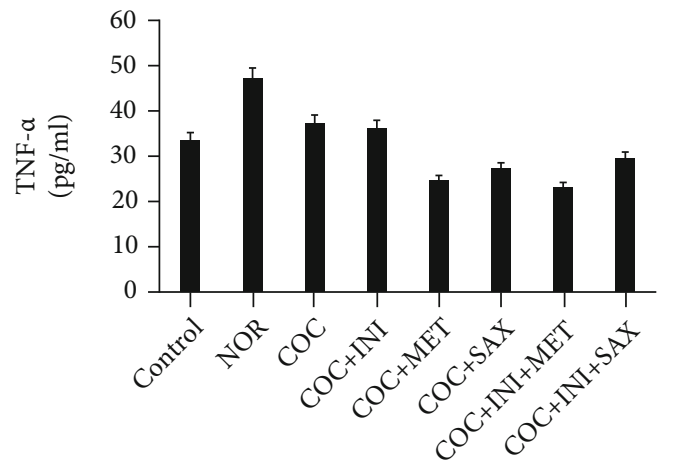

(a)

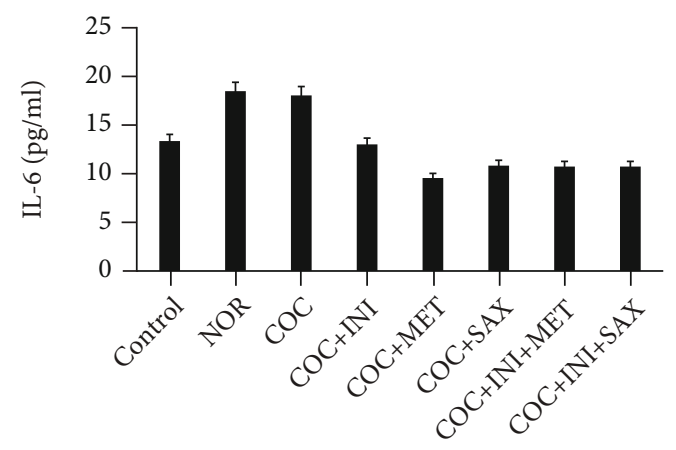

(b)

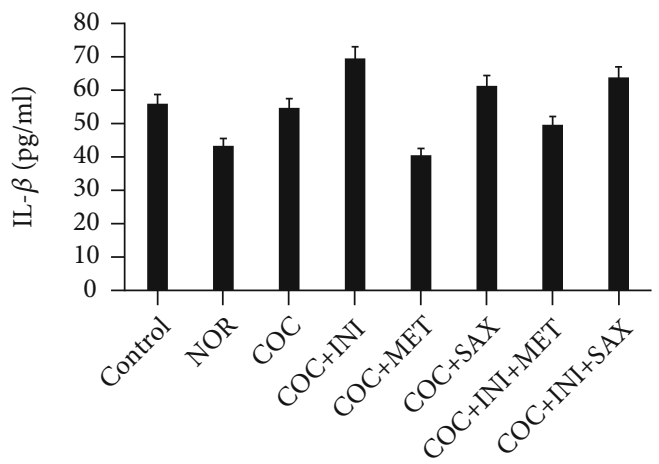

(c)

Figure 7: $(\mathrm{a}-\mathrm{c})$ No statistically significant alterations across the test groups for the inflammatory mediators: TNF- $\alpha$, IL-6, and IL-1 $\beta$, respectively. However, there is a nonstatistical increase in the level of TNF- $\alpha$ and IL- 6 in the COC group and a subsequent reduction in the MET and INI+MET groups for both mediators, $p \leq 0.05$; mean \pm SEM, $n=6$.

to the pancreas, since this is not insulin insufficiency as seen in the etiology of type 1 diabetes mellitus [56]. Organosomatic index is a more definitive examination of organ weight which shows the proportional sizes of organs and depicts the status of organ systems. Organ sizes relative to body weight may change faster than organism weight and are an important indicator of organ toxicity. Organosomatic indices have been globally accepted as a pointer of organ-system integrity of animals [57].
High blood glucose (hyperglycemia) and hyperinsulinemia are major features of metabolic syndrome and particular risk factors in type 2 diabetes mellitus [50]. Use of combined oral contraceptive induces significant increases in both fasting blood glucose and insulin levels; this establishes the fact that the continuous use of this contraceptive predisposes to type 2 diabetes mellitus and cardiovascular dysfunctions. Treatment with saxagliptin markedly reduced the fasting blood glucose levels, and a reduction in glycemic 


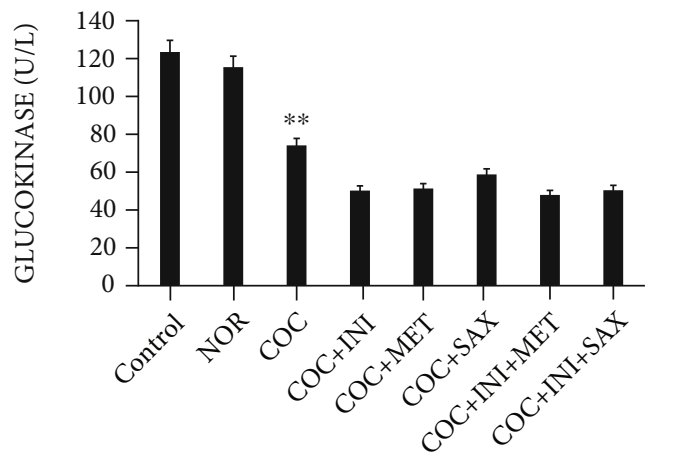

(a)

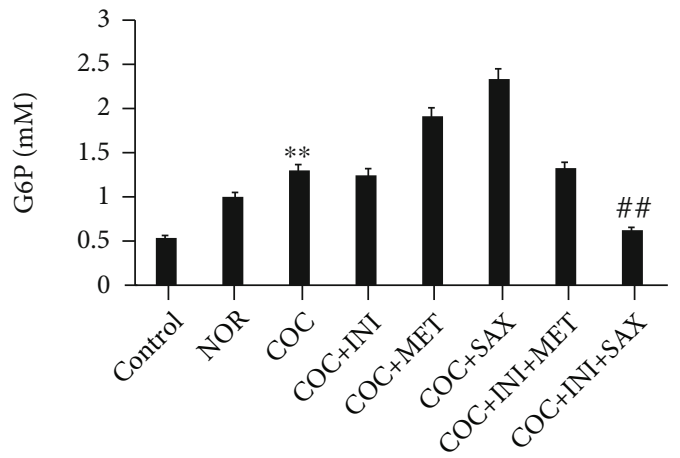

(b)

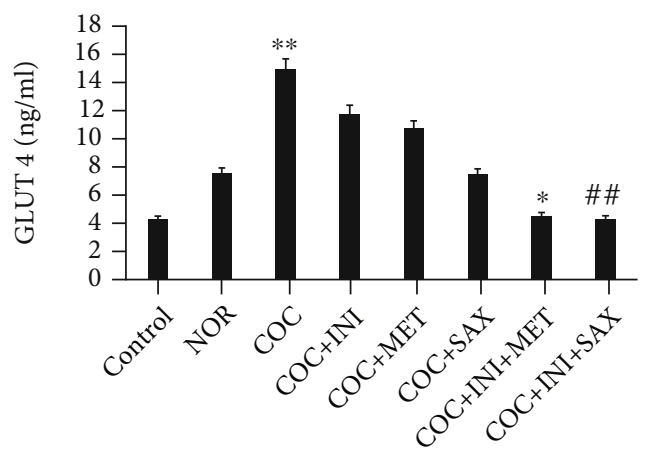

(c)

FIgURE 8: (a) COC caused a significant decrease in glucokinase level when compared with control $\left({ }^{* *} p \leq 0.01\right)$; this decrease was not reversed by any of the treatment group $(p \leq 0.01)$ when compared with the COC group. (b) COC increased the G6P level $\left({ }^{* *} p \leq 0.01\right)$; this effect was reversed significantly by the INI+SAX group $\left({ }^{\# \#} p \leq 0.01\right)$. (c) COC increased the GLUT4 levels significantly $\left({ }^{* *} p \leq 0.01\right)$; this effect was reversed in INI+MET $\left({ }^{*} p \leq 0.05\right)$ and INI+SAX $\left({ }^{\# \#} p \leq 0.01\right)($ mean \pm SEM, $n=6)$.

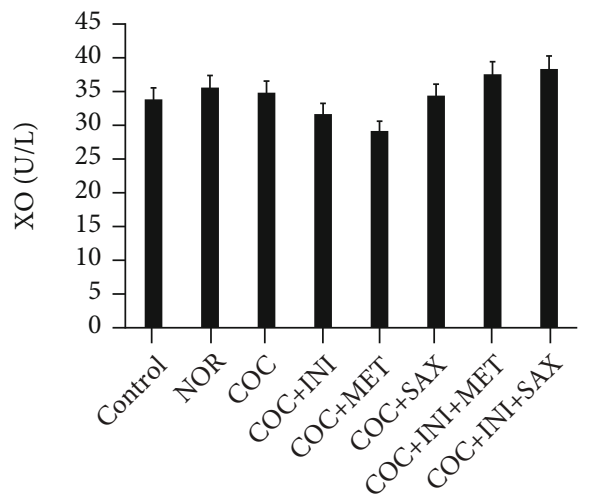

(a)

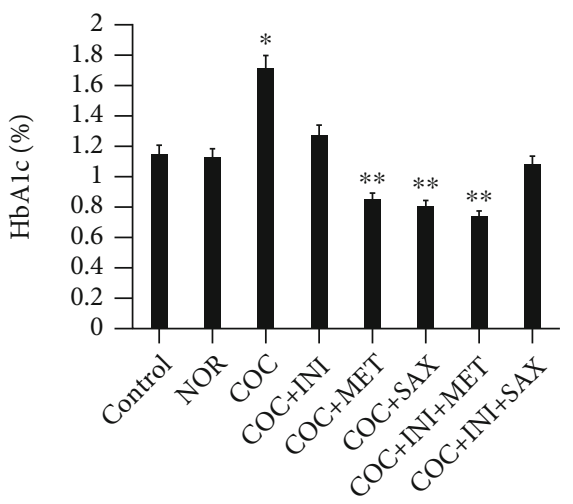

(b)

FIgURE 9: (a) No significant alterations across the test groups for xanthine oxidase (XO), $p \leq 0.05$; (b) COC increased significantly the level of HBA1c when compared with the control $\left({ }^{*} p \leq 0.05\right)$; this effect was reversed significantly in the MET, SAX, and INI+MET groups $\left({ }^{* *} p \leq 0.01\right)$; mean \pm SEM, $n=6 ; n=8$.

level was also observed in the intranasal insulin group. Insulin resistance depicted by hyperinsulinemia can dysregulate glucose metabolism generating chronic hyperglycemia, leading to the onset of cardiac related abnormalities [58]. A proper management of the elements of MetS in women of reproductive age can prevent the development of cardiovascular diseases, which is the global leading cause of death [59]. C-reactive peptide, C-peptide, and ADA levels were not significantly affected; also, the oral glucose tolerance test was not affected by the combined oral contraceptives.

Lipid profile analysis, with particular focus on the HDL, LDL, and triglyceride level, has been specifically linked to metabolic syndrome [60]. Combined oral contraceptive use reduced the level of HDL; this reduction was reversed by intranasal insulin and saxagliptin combined therapy; LDL and total cholesterol levels were reduced by the administration of 
norethindrone; however, triglyceride levels were not affected by the combined contraceptives. Insulin resistance alters systemic lipid metabolism leading to the development of dyslipidemia [61]; the 3 popular lipid-related aberrations are as follows: (1) upregulated levels of plasma triglycerides, (2) low levels of high-density lipoprotein, and (3) the presence of low-density lipoproteins. These features, in combination with dysfunction of the endothelial, which can also be caused by disrupted insulin signaling, are important underlying factors to formation of atherosclerotic plaques [58].

The association between oxidative stress and insulin resistance has been reported to be a predisposing link to cardiovascular diseases [62]. Pancreatic homogenate antioxidant assay showed that the level of catalase enzyme was significantly reduced consequent upon exposure to combined oral contraceptives; this reduction was reversed by treatment with intranasal insulin and metformin combination therapy. Catalase is an antioxidant enzyme which catalyzes the breakdown of hydrogen peroxide to water and oxygen. It is crucial for the protection of cells from oxidative damage by reactive oxygen species (ROS) [63]. Deficiency or malfunction of catalase is postulated to be related to the pathogenesis of many age-associated degenerative diseases like diabetes mellitus, hypertension, anemia, and vitiligo [64]. Other antioxidant parameters such as SOD, GSH, GPx, and MDA levels were not affected significantly by treatment with the combined oral contraceptive in this study.

Glycated hemoglobin (HbAlc) levels have been used as a diagnostic parameter for profiling insulin resistance in patients and a probe for type 2 diabetes mellitus [65]. We checked for the effect of combined oral contraceptive treatment on HbAlc and discovered a significantly high level $(p \leq 0.05)$; this effect was reversed in the MET, SAX, and INI+SAX groups $(p \leq 0.01)$, suggesting their efficacy in the management of MetS caused by the use of combined oral contraceptive which could subsequently lead to the prevention of type $2 \mathrm{DM}$ and cardiotoxicity. The formation of the sugar-hemoglobin linkage indicates the presence of excessive sugar in the bloodstream, often indicative of diabetes [66]. $\mathrm{HbA1C}$ is of particular interest because it is easy to detect. Liver GLUT4 level was upregulated in the COC group and subsequently reversed in the INI+MET and INI+SAX groups. However, we suggest this could be as a result of the length of the study; we expected a reduction in the level of GLUT4 activity. During the normal physiology of glucose-stimulated secretion of insulin, beta-cells release insulin into circulation, activating GLUT4-dependent glucose uptake into peripheral tissue. Glucokinase level was significantly reduced in the COC group; glucose-6-phosphate levels were increased in the same group but subsequently reduced in the INI+SAX group. Glucokinase converts glucose to glucose-6-phosphate which may be converted to glycogen for storage [67]. Inflammatory cytokines such as TNF- $\alpha$, IL- 6 , and IL- $1 \beta$ play significant roles in the etiology of metabolic syndrome. Scientific evidence suggests that obesity, in particular chronic visceral adiposity, is associated with inflammatory markers including interleukin-6 (IL-6) and tumor necrosis factor-alpha (TNF- $\alpha$ ) [68]. Studies have shown that IL- 6 and TNF- $\alpha$ are secreted from infiltrated macrophages into adipose tissue and have critical roles in MetS, insulin resistance, nonalcoholic fatty liver disease, and atherogenesis [69]. Our study showed that these cytokines (TNF- $\alpha$ and IL-6) were affected without statistical significance. Nonstatistically significant increase in TNF- $\alpha$ and in COC-treated group was reversed by administration of MET and combined INI+MET. These suggest an early stage or development of MetS can be arrested with these therapeutic regimens.

\section{Conclusion}

Findings from this study show that chronic combined oral contraceptive treatment predisposes female albino rats to metabolic syndrome which features hyperglycemia, dyslipidemia, hyperinsulinemia, etc., all of which were featured in this present study. The use combination therapies particularly involving saxagliptin and metformin corrected some of these metabolic abnormalities that have a direct link to glucose metabolism. Thus, the metabolic syndrome status of combined oral contraceptive users should be closely monitored, and therapeutic interventions should be employed to prevent development of type $2 \mathrm{DM}$ and other cardiovascular pathologies. A clinical study should be engaged in order to establish the translational relevance of these findings in women of reproductive age.

\section{Data Availability}

The datasets used and/or analyzed during the current study are available from the corresponding author upon request.

\section{Conflicts of Interest}

The authors affirm that they have no conflict of interest. All authors affirm they have received no financial or other supports, from any organization that could have influenced the submitted work.

\section{Authors' Contributions}

All authors made significant contributions to conception and design, acquisition of the data, or analysis and development of the manuscript. SOA and OO conceptualized the work and worked extensively on the development of the manuscript. SOA, FJ, and OA carried out the laboratory work including providing expertise in the methods used and the overall inference from the findings of the research work. All authors read and approved the final manuscript.

\section{Acknowledgments}

We thank Pharmacist Chloe Fatigun and Mr. Benjamin Ejimkonye for their technical support during the research.

\section{References}

[1] K. Daniels and J. C. Abma, Current Contraceptive Status among Women Aged 15-49: United States, 2015-2017, National 
Center for Health Statistics, 2018, https://www.cdc.gov/nchs/ products/databriefs/db388.html.

[2] L. S. Mørch, C. W. Skovlund, P. C. Hannaford, L. Iversen, S. Fielding, and $\varnothing$. Lidegaard, "Contemporary hormonal contraception and the risk of breast cancer," The New England Journal of Medicine, vol. 377, no. 23, pp. 2228-2239, 2017.

[3] R. Sitruk-Ware and A. Nath, "Metabolic effects of contraceptive steroids," Reviews in Endocrine \& Metabolic Disorders, vol. 12, no. 2, pp. 63-75, 2011.

[4] D. B. Cooper and H. Mahdy, Oral Contraceptive Pills, StatPearls, 2020.

[5] K. M. Curtis, N. K. Tepper, T. C. Jatlaoui et al., "US medical eligibility criteria for contraceptive use, 2016," Morbidity and Mortality Weekly Report, vol. 65, no. 3, pp. 1-3, 2016.

[6] J. Y. Jeong, M. K. Kim, I. Lee et al., "Polycystic ovarian morphology is associated with primary dysmenorrhea in young Korean women," Obstetrics \& Gynecology Science, vol. 62, no. 5, p. 329, 2019.

[7] V. de Leo, M. C. Musacchio, V. Cappelli, P. Piomboni, and G. Morgante, "Hormonal contraceptives: pharmacology tailored to women's health," Human Reproduction Update, vol. 22, no. 5, pp. 634-646, 2016.

[8] S. Manzoor, M. A. Ganie, S. Majid et al., "Analysis of intrinsic and extrinsic coagulation pathway factors in OCP treated PCOS women," Indian Journal of Clinical Biochemistry, vol. 17, pp. 1-10, 2020.

[9] Q. Wang, P. Würtz, K. Auro et al., "Effects of hormonal contraception on systemic metabolism: cross-sectional and longitudinal evidence," International Journal of Epidemiology, vol. 45, no. 5, pp. 1445-1457, 2016.

[10] S. Panicker, S. Mann, J. Shawe, and J. Stephenson, "Evolution of extended use of the combined oral contraceptive pill," The Journal of Family Planning and Reproductive Health Care, vol. 40, no. 2, pp. 133-141, 2014.

[11] A. Edelman, M. F. Gallo, M. D. Nichols, J. T. Jensen, K. F. Schulz, and D. A. Grimes, "Continuous versus cyclic use of combined oral contraceptives for contraception: systematic Cochrane review of randomized controlled trials," Human Reproduction, vol. 21, no. 3, pp. 573-578, 2006.

[12] F. de Castro Coelho and C. Barros, "The potential of hormonal contraception to influence female sexuality," The Journal of Reproductive Medicine, vol. 2019, article 9701384, pp. 1-9, 2019.

[13] F. Z. Stanczyk, D. F. Archer, and B. R. Bhavnani, "Ethinyl estradiol and $17 \beta$-estradiol in combined oral contraceptives: pharmacokinetics, pharmacodynamics and risk assessment," Contraception, vol. 87, no. 6, pp. 706-727, 2013.

[14] S. Christin-Maitre, "Use of hormone replacement in females with endocrine disorders," Hormone Research in Padiatrics, vol. 87, no. 4, pp. 215-223, 2017.

[15] N. Santoro, R. Worsley, K. K. Miller, S. J. Parish, and S. R. Davis, "Role of estrogens and estrogen-like compounds in female sexual function and dysfunction," The Journal of Sexual Medicine, vol. 13, no. 3, pp. 305-316, 2016.

[16] H. Kuhl, "Pharmacology of estrogens and progestogens: influence of different routes of administration," Climacteric, vol. 8, Suppplement 1, pp. 3-63, 2005.

[17] G. Evans and E. L. Sutton, "Oral contraception," Medicina Clínica, vol. 99, no. 3, pp. 479-503, 2015.

[18] K. K. Scarsi, K. M. Darin, S. Nakalema et al., "Unintended pregnancies observed with combined use of the levonorgestrel contraceptive implant and efavirenz-based antiretroviral therapy: a three-arm pharmacokinetic evaluation over 48 weeks," Clinical Infectious Diseases, vol. 62, no. 6, pp. 675-682, 2016.

[19] R. Peck, W. Rella, J. Tudela, J. Aznar, and B. Mozzanega, “Does levonorgestrel emergency contraceptive have a postfertilization effect? A review of its mechanism of action," The Linacre Quarterly, vol. 83, no. 1, pp. 35-51, 2016.

[20] C. Kahlenborn, R. Peck, and W. B. Severs, "Mechanism of action of levonorgestrel emergency contraception," The Linacre Quarterly, vol. 82, no. 1, pp. 18-33, 2015.

[21] J. Elks, Ed., The Dictionary of Drugs: Chemical Data: Chemical Data, Structures and Bibliographies, Springer, 2014.

[22] L. Li, R. Zhang, J. Zeng et al., "Effectiveness and safety assessment of drospirenone/ethinyl estradiol tablet in treatment of PCOS patients: a single center, prospective, observational study," BMC Women's Health, vol. 20, no. 1, pp. 1-7, 2020.

[23] A. S. De Melo, R. Reis, R. A. Ferriani, and C. Vieira, "Hormonal contraception in women with polycystic ovary syndrome: choices, challenges, and non-contraceptive benefits," Open Access Journal of Contraception, vol. Volume 8, pp. 1323, 2017.

[24] I. J. Halperin, S. Sujana Kumar, D. F. Stroup, and S. E. Laredo, "The association between the combined oral contraceptive pill and insulin resistance, dysglycemia and dyslipidemia in women with polycystic ovary syndrome: a systematic review and meta-analysis of observational studies," Human Reproduction, vol. 26, no. 1, pp. 191-201, 2011.

[25] O. A. Adeyanju, O. A. Soetan, A. O. Soladoye, and L. A. Olatunji, "Oral hormonal therapy with ethinylestradiol-levonorgestrel improves insulin resistance, obesity, and glycogen synthase kinase- 3 independent of circulating mineralocorticoid in estrogen-deficient rats," Canadian Journal of Physiology and Pharmacology, vol. 96, no. 6, pp. 577-586, 2018.

[26] P. Rorsman and M. O. Huising, "The somatostatin-secreting pancreatic $\delta$-cell in health and disease," Nature Reviews. Endocrinology, vol. 14, no. 7, pp. 404-414, 2018.

[27] P. V. Röder, B. Wu, Y. Liu, and W. Han, "Pancreatic regulation of glucose homeostasis," Experimental \& Molecular Medicine, vol. 48, no. 3, article e219, 2016.

[28] J. Myers, P. Kokkinos, and E. Nyelin, "Physical activity, cardiorespiratory fitness, and the metabolic syndrome," Nutrients, vol. 11, no. 7, p. 1652, 2019.

[29] K. D. Bruce and F. R. Cagampang, "Epigenetic priming of the metabolic syndrome," Toxicology Mechanisms and Methods, vol. 21, no. 4, pp. 353-361, 2011.

[30] G. Ricci, I. Pirillo, D. Tomassoni, A. Sirignano, and I. Grappasonni, "Metabolic syndrome, hypertension, and nervous system injury: epidemiological correlates," Clinical and Experimental Hypertension, vol. 39, no. 1, pp. 8-16, 2017.

[31] M. D. DeBoer, M. J. Gurka, S. H. Golden et al., "Independent associations between metabolic syndrome severity and future coronary heart disease by sex and race," Journal of the American College of Cardiology, vol. 69, no. 9, pp. 1204-1205, 2017.

[32] K. Ganesan and S. Sultan, Oral Hypoglycemic Medications, StatPearls, 2019.

[33] J. Grisouard, K. Timper, T. M. Radimerski et al., "Mechanisms of metformin action on glucose transport and metabolism in human adipocytes," Biochemical Pharmacology, vol. 80, no. 11, pp. 1736-1745, 2010.

[34] S. Correia, C. Carvalho, M. S. Santos et al., "Metformin protects the brain against the oxidative imbalance promoted by 
type 2 diabetes," Medicinal Chemistry, vol. 4, no. 4, pp. 358364, 2008.

[35] P. Sa-nguanmoo, P. Tanajak, S. Kerdphoo et al., "SGLT2inhibitor and DPP-4 inhibitor improve brain function via attenuating mitochondrial dysfunction, insulin resistance, inflammation, and apoptosis in HFD-induced obese rats," Toxicology and Applied Pharmacology, vol. 333, pp. 43-50, 2017.

[36] National Research Council, Sustainability and the US EPA, National Academies Press, 2011.

[37] U. Albus, "Guide for the care and use of laboratory animals (8th edn)," Laboratory Animals, vol. 46, no. 3, pp. 267-268, 2012.

[38] A. A. Njan, C. O. Fatigun, A. M. Alli-Oluwafuyi et al., "Effect of intranasal insulin on peripheral glucose profile in dexamethasone-induced insulin resistance in Wistar rats," Beni-Suef University Journal of Basic and Applied Sciences, vol. 7, no. 4, pp. 516-524, 2018.

[39] D. E. Wilson and M. J. Spiger, "A dual precipitation method for quantitative plasma lipoprotein measurement without ultracentrifugation," The Journal of Laboratory and Clinical Medicine, vol. 82, no. 3, pp. 473-582, 1973.

[40] A. Hafiane and J. Genest, "High density lipoproteins: measurement techniques and potential biomarkers of cardiovascular risk," BBA Clinical, vol. 3, pp. 175-188, 2015.

[41] P. Rao and T. N. Pattabiraman, "Further studies on the mechanism of phenol-sulfuric acid reaction with furaldehyde derivatives," Analytical Biochemistry, vol. 189, no. 2, pp. 178-181, 1990.

[42] O. H. Lowry, N. J. Rosebrough, A. L. Farr, and R. J. Randall, "Protein measurement with the Folin phenol reagent," The Journal of Biological Chemistry, vol. 193, no. 1, pp. 265-275, 1951.

[43] S. Marklund and G. Marklund, "Involvement of the superoxide anion radical in the autoxidation of pyrogallol and a convenient assay for superoxide dismutase," European Journal of Biochemistry, vol. 47, no. 3, pp. 469-474, 1974.

[44] J. T. Rotruck, A. L. Pope, H. E. Ganther, A. B. Swanson, D. G. Hafeman, and W. G. Hoekstra, "Selenium: biochemical role as a component of glutathione peroxidase," Science, vol. 179, no. 4073 , pp. 588-590, 1973.

[45] A. K. Sinha, "Colorimetric assay of catalase," Analytical Biochemistry, vol. 47, no. 2, pp. 389-394, 1972.

[46] S. S. Mohamed Isa, A. Ablat, and J. Mohamad, "The antioxidant and xanthine oxidase inhibitory activity of Plumeria rubra flowers," Molecules, vol. 23, no. 2, p. 400, 2018.

[47] A. U. Kabir, M. B. Samad, A. Ahmed et al., "Aqueous fraction of Beta vulgaris ameliorates hyperglycemia in diabetic mice due to enhanced glucose stimulated insulin secretion, mediated by acetylcholine and GLP-1, and elevated glucose uptake via increased membrane bound GLUT4 transporters," PLoS One, vol. 10, no. 2, article e0116546, 2015.

[48] D. E. Moller and K. D. Kaufman, "Metabolic syndrome: a clinical and molecular perspective," Annual Review of Medicine, vol. 56, no. 1, pp. 45-62, 2005.

[49] M. G. Saklayen, "The global epidemic of the metabolic syndrome," Current Hypertension Reports, vol. 20, no. 2, p. 12, 2018.

[50] M. K. Lee, K. Han, M. K. Kim et al., "Changes in metabolic syndrome and its components and the risk of type 2 diabetes: a nationwide cohort study," Scientific Reports, vol. 10, no. 1, p. 2313,2020 .
[51] S. C. Marott, B. G. Nordestgaard, A. Tybjærg-Hansen, and M. Benn, "Components of the metabolic syndrome and risk of type 2 diabetes," The Journal of Clinical Endocrinology and Metabolism, vol. 101, no. 8, pp. 3212-3221, 2016.

[52] M. B. Schulze, "Metabolic health in normal-weight and obese individuals," Diabetologia, vol. 62, no. 4, pp. 558-566, 2019.

[53] M. Gao, J. Lv, C. Yu et al., "Metabolically healthy obesity, transition to unhealthy metabolic status, and vascular disease in Chinese adults: a cohort study," PLoS Medicine, vol. 17, no. 10, article e1003351, 2020.

[54] A. Lukács, E. Horváth, Z. Máté et al., “Abdominal obesity increases metabolic risk factors in non-obese adults: a Hungarian cross-sectional study," BMC Public Health, vol. 19, no. 1, 2019.

[55] A. C. Ghezzi, L. T. Cambri, J. D. Botezelli, C. Ribeiro, R. A. Dalia, and M. A. de Mello, "Metabolic syndrome markers in Wistar rats of different ages," Diabetology and Metabolic Syndrome, vol. 4, no. 1, p. 16, 2012.

[56] M. Wolosowicz, B. Lukaszuk, and A. Chabowski, "The causes of insulin resistance in type 1 diabetes mellitus: is there a place for quaternary prevention?," International Journal of Environmental Research and Public Health, vol. 17, no. 22, p. 8651, 2020.

[57] N. Gupta, D. K. Gupta, and P. K. Sharma, "Condition factor and organosomatic indices of parasitized Rattus rattus as indicators of host health," Journal of Parasitic Diseases, vol. 41, no. 1, pp. 21-28, 2017.

[58] V. Ormazabal, S. Nair, O. Elfeky, C. Aguayo, C. Salomon, and F. A. Zuñiga, "Association between insulin resistance and the development of cardiovascular disease," Cardiovascular Diabetology, vol. 17, no. 1, pp. 1-4, 2018.

[59] L. Kazlauskienè, J. Butnorienė, and A. Norkus, "Metabolic syndrome related to cardiovascular events in a 10-year prospective study," Diabetology and Metabolic Syndrome, vol. 7, no. 1, p. 102, 2015.

[60] A. Tahir, P. J. Martinez, F. Ahmad et al., "An evaluation of lipid profile and pro-inflammatory cytokines as determinants of cardiovascular disease in those with diabetes: a study on a Mexican American cohort," Scientific Reports, vol. 11, no. 1, p. 2435, 2021.

[61] D. M. Erion, H. J. Park, and H. Y. Lee, "The role of lipids in the pathogenesis and treatment of type 2 diabetes and associated co-morbidities," BMB Reports, vol. 49, no. 3, pp. 139-148, 2016.

[62] S. Dzięgielewska-Gęsiak, D. Stołtny, A. Brożek, M. MucWierzgoń, and E. Wysocka, "Are insulin-resistance and oxidative stress cause or consequence of aging," Experimental Biology and Medicine, vol. 245, no. 14, pp. 1260-1267, 2020.

[63] P. Chelikani, I. Fita, and P. C. Loewen, "Diversity of structures and properties among catalases," Cellular and Molecular Life Sciences, vol. 61, no. 2, pp. 192-208, 2004.

[64] A. Nandi, L. J. Yan, C. K. Jana, and N. Das, "Role of Catalase in Oxidative Stress- and Age-Associated Degenerative Diseases," Oxidative Medicine and Cellular Longevity, vol. 2019, Article ID 9613090, 19 pages, 2019.

[65] G. Saravia, F. Civeira, Y. Hurtado-Roca et al., "Glycated hemoglobin, fasting insulin and the metabolic syndrome in males. Cross-sectional analyses of the Aragon workers health study baseline," PLoS One, vol. 10, no. 8, article e0132244, 2015.

[66] S. I. Sherwani, H. A. Khan, A. Ekhzaimy, A. Masood, and M. K. Sakharkar, "Significance of HbA1c test in diagnosis and 
prognosis of diabetic patients," Biomarker Insights, vol. 11, pp. BMI.S38440-BMI.S38104, 2016.

[67] N. B. Whitticar and C. S. Nunemaker, "Reducing glucokinase activity to enhance insulin secretion: a counterintuitive theory to preserve cellular function and glucose homeostasis," Frontiers in Endocrinology, vol. 11, p. 378, 2020.

[68] M. Mohammadi, M. H. Gozashti, M. Aghadavood, M. R. Mehdizadeh, and M. M. Hayatbakhsh, "Clinical significance of serum IL-6 and TNF- $\alpha$ levels in patients with metabolic syndrome," Reports of Biochemistry \& Molecular Biology, vol. 6, no. 1, pp. 74-79, 2017.

[69] F. Wang and Z. Mo, "NLRP3 inflammasome in metabolic syndrome," Brazilian Journal of Pharmaceutical Sciences, vol. 26, p. 56, 2021. 